\title{
Epicyclic orbits in a viscous fluid about a precessing rod: Theory and experiments at the micro- and macro-scales
}

\author{
Elizabeth L. Bouzarth, ${ }^{1}$ Adam Brooks, ${ }^{1}$ Roberto Camassa, ${ }^{1}$ Hao Jing, ${ }_{1}^{2}$ Terry Jo Leiterman, ${ }^{1}$ Richard M. McLaughlin, ${ }^{1, *}$ \\ Richard Superfine, ${ }^{3, \dagger}$ Jonathan Toledo, ${ }^{1}$ and Leandra Vicci ${ }^{4}$ \\ ${ }^{1}$ Department of Mathematics, University of North Carolina, Chapel Hill, North Carolina 27599-3250, USA \\ ${ }^{2}$ Department of Applied and Material Sciences, University of North Carolina, Chapel Hill, North Carolina 27599-3250, USA \\ ${ }^{3}$ Department of Physics and Astronomy, University of North Carolina, Chapel Hill, North Carolina 27599-3250, USA \\ ${ }^{4}$ Department of Computer Science, University of North Carolina, Chapel Hill, North Carolina 27599-3250, USA
}

(Received 4 May 2006; revised manuscript received 27 December 2006; published 25 July 2007)

\begin{abstract}
We present experimental observations and quantified theoretical predictions of the nanoscale hydrodynamics induced by nanorod precession emulating primary cilia motion in developing embryos. We observe phenomena including micron size particles which exhibit epicyclic orbits with coherent fluctuations distinguishable from comparable amplitude thermal noise. Quantifying the mixing and transport physics of such motions on small scales is critical to understanding fundamental biological processes such as extracellular redistribution of nutrients. We present experiments designed to quantify the trajectories of these particles, which are seen to consist of slow orbits about the rod, with secondary epicycles quasicommensurate with the precession rate. A first-principles theory is developed to predict trajectories in such time-varying flows. The theory is further tested using a dynamically similar macroscale experiment to remove thermal noise effects. The excellent agreement between our theory and experiments confirms that the continuum hypothesis applies all the way to the scales of such submicron biological motions.
\end{abstract}

DOI: 10.1103/PhysRevE.76.016313 PACS number(s): 47.61.-k, 47.63.mf, 47.54.Bd, 47.54.De

\section{INTRODUCTION}

Fueled by the recent advances of nanoscale technology in biophysics, there is renewed interest in the fundamental physics at the borderline between molecular and continuum scales in microscopic processes. The motion and hydrodynamic response of microscale bodies moving through fluids is a challenging problem in dynamical microbiology.

Examples of such interactions include the role which primary cilia play in the establishment of right-left asymmetry in developing embryos $[1,2]$, mucociliary transport in the lung [3,4], and the propulsion of flagellated organisms [5]. Controlled experimental observations on these small scales is difficult because of the need to perform repeatable motions of nanoscale structures. The engineering of devices that can generate controlled motion of these structures is therefore of crucial importance. For a predictive understanding of these microscopic flows, it is equally crucial to develop a firstprinciples theory that predicts the detailed features of the resulting flows. Given microbiological scales, a starting point for developing such theory is the Stokes equations. However, in strongly fluctuating system on such scales, the precise boundary conditions are currently under investigation and in particular the validity of the no-slip condition for rigid bodies on these scales $[6,7]$. In the present study, we observe phenomena which we document experimentally and theoretically, and in the process we document that the no-slip condition appears valid for the geometries and time scales in our experiments.

The geometry in the present study is set by a precessing rod sweeping an upright cone above a flat, no slip, plane.

\footnotetext{
*rmm@email.unc.edu

†rsuper@physics.unc.edu
}

This prescribed rod precession induces motion in the surrounding fluid. Nanoscale measurements are performed using soft magnetic nanorods 20 microns in length by 200 nanometers in cross-sectional diameter actuated at $300 \mathrm{rpm}$ $(5 \mathrm{~Hz})$ in water using a custom built magnetic force system integrated with an optical microscope. The observations involve the motion of small passive particles which exhibit slow orbits around the precessing rod, and a fast, roughly precession-rate commensurate epicycle motion. The amplitude of this fast epicycle admits strong variation with distance from the precessing rod. We present an asymptotic solution to the Stokes equations in this geometry which is developed using a family of fundamental singularities and images introduced by Blake [8]. Using slender body theory [9], we asymptotically solve the relevant integral equation to determine the singularity strengths and obtain a mathematical solution which can be used to predict the amplitude of this epicycle. To study the details of these solutions and the issue of dynamical similarity across four orders of magnitude in length scale we have performed tabletop (macroscale) control measurements using a steel pin $1 \mathrm{~cm}$ long, by $1 \mathrm{~mm}$ cross-sectional diameter precessing at approximately $9 \mathrm{rpm}$ in a high viscosity solution. The macroscale measurements quantitatively validate the mathematical theory using no adjustable parameters in an environment free from thermal fluctuations. The mathematical theory is predictive in the near field on the microscale measurements, but deteriorates at far field. Discussion is given regarding this lack of agreement at far field in the microscale as regards uncertainty in the measurements and random thermal fluctuations. In the following, we first summarize our theory of precessing rod fluid-structure interactions, followed by its detailed validation in the macroscale experiments and comparison with the nanoscale fluid flows. 


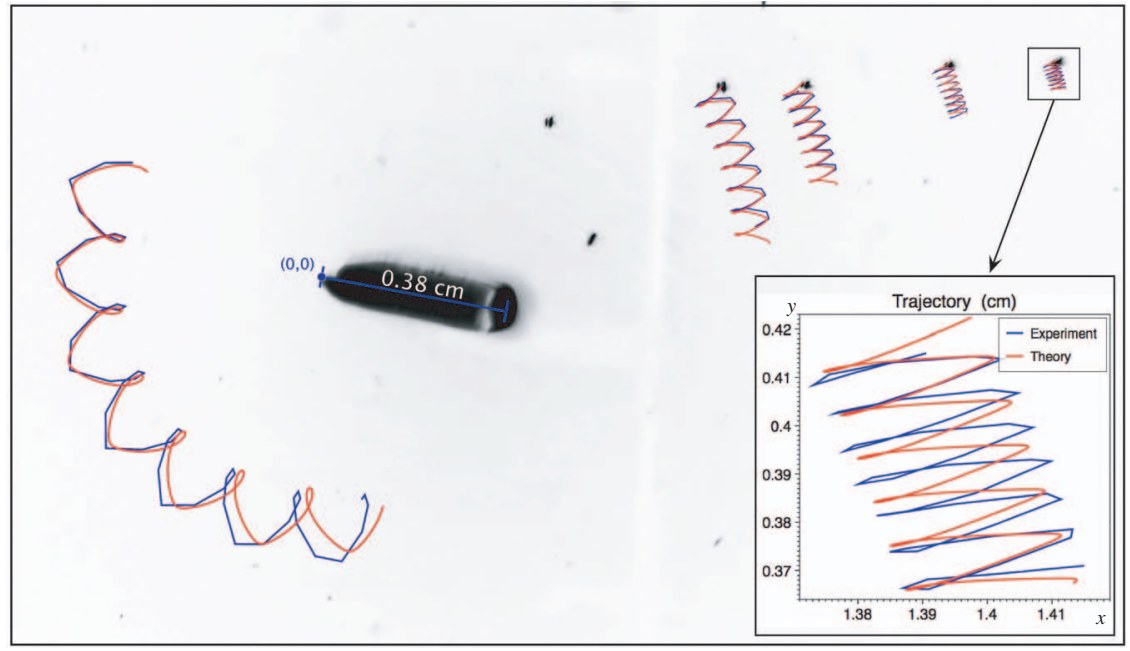

FIG. 1. (Color) Theoretical trajectories compared to bubble trajectories from a macroscale experiment. The rod base is at the origin and the black dots are the tracked bubbles. Trajectories shown are initially at 0.61 to $0.65 \mathrm{~cm}$ from the floor of the tank. The rod length is $0.76 \mathrm{~cm}$, the cone half-angle is $30^{\circ}$, the cone radius $R_{c}=0.38 \mathrm{~cm}$, the cone height $H_{c}=0.66 \mathrm{~cm}$, the rod diameter is $0.9 \mathrm{~mm}$, and the rod rotates clockwise at 9 RPM. Theoretical trajectories are computed using a fourth-order Runge-Kutta solver with $10^{4}$ time steps per revolution for $\dot{\mathbf{x}}=\mathbf{u}(\mathbf{x}, t)$. The theoretical trajectories use the stated cone geometry and rod radius, and initial position $\left(x_{0}, y_{0}\right)$ in the plane provided by the experiment. The initial vertical position is set at $z_{0}=0.625 \mathrm{~cm}$ for each simulated trajectory.

\section{HYDRODYNAMIC SOLUTION}

The fundamental governing equations for an incompressible fluid system are given by the unsteady, nonlinear NavierStokes equations. The Reynolds number $\operatorname{Re}=\ell \mathbf{U} / \nu$ is a nondimensional number measuring the ratio of inertial forces relative to viscous forces where $\ell, \mathbf{U}$, and $\nu$ are characteristic length, velocity, and viscosity scales in the fluid system under consideration. When the Reynolds number is small, the nonlinearity in the Navier-Stokes equations can be neglected.

Consider a slender body of radius $r_{0}$ and length $L$ attached to a no-slip plane sweeping out a cone of angle $\kappa$ in a viscous fluid at a rate of $\omega$ such that the resulting Reynolds number is small. For $L$ and $\omega$ finite and large fluid viscosities $\nu$, a nondimensionalization of the Navier-Stokes equations provides that the governing equations for this motion are the linear, steady Stokes equations $\nabla^{2} \mathbf{u}(\mathbf{x})-\nabla p=0, \boldsymbol{\nabla} \cdot \mathbf{u}=0$ with boundary conditions depending upon the cone angle $\kappa$.

In 1970, Batchelor [9] used slender body theory to construct solutions to Stokes equations for bodies of arbitrary cross section embedded in uniform and certain linear flows. These solutions were constructed by placing Stokeslets along the interior centerline of the body. A Stokeslet [10] is the primary fundamental solution to Stokes equations and is due to a point force of strength $\boldsymbol{\alpha}$ applied to the flow field. In 1971, Blake [8] constructed a Green's function for Stokes equations in the presence of a no-slip plane, $\mathbf{u}(z=0)=0$, using the method of images. The resulting image system consists of a Stokeslet along with higher order derivatives of this singularity.

By utilizing the fundamental solution of Blake and the slender body theory of Batchelor, we have constructed an asymptotic solution for a slender body attached to the no-slip plane $z=0$ tilted by an angle $\kappa$ from the positive vertical axis sweeping out a cone. Slenderness is defined through $\delta$ $=r_{0} / L \ll 1$. The velocity field $\mathbf{u}(\mathbf{x})=R \mathbf{v}\left(R^{T} \mathbf{x}\right)[11-13]$ that generates the particle trajectories shown in Fig. 1 is given as a time-dependent rotation, $R$, about the vertical axis, of the velocity field

$$
\begin{aligned}
\mathbf{v}(\mathbf{x})= & \int_{0}^{L}\left[\left(\frac{\boldsymbol{\alpha}(s)}{|\mathbf{x}-\mathbf{s}|}+\frac{(\mathbf{x}-\mathbf{s})[(\mathbf{x}-\mathbf{s}) \cdot \boldsymbol{\alpha}(s)]}{|\mathbf{x}-\mathbf{s}|^{3}}\right)\right. \\
& -\left(\frac{\boldsymbol{\alpha}(s)}{\left|\mathbf{x}-\mathbf{s}^{*}\right|}+\frac{\left(\mathbf{x}-\mathbf{s}^{*}\right)\left[\left(\mathbf{x}-\mathbf{s}^{*}\right) \cdot \boldsymbol{\alpha}(s)\right]}{\left|\mathbf{x}-\mathbf{s}^{*}\right|^{3}}\right) \\
& +2 s \cos \kappa\left(\frac{-z \boldsymbol{\alpha}(s)+\widehat{\mathbf{e}_{z}\left[\left(\mathbf{x}-\mathbf{s}^{*}\right) \cdot \boldsymbol{\alpha}(s)\right]}}{\left|\mathbf{x}-\mathbf{s}^{*}\right|^{3}}\right. \\
& \left.\left.+\frac{3 z\left(\mathbf{x}-\mathbf{s}^{*}\right)\left[\left(\mathbf{x}-\mathbf{s}^{*}\right) \cdot \boldsymbol{\alpha}(s)\right]}{\left|\mathbf{x}-\mathbf{s}^{*}\right|^{5}}\right)\right] d s,
\end{aligned}
$$

where $\widehat{\mathbf{e}_{z}}=(0,0,1), \quad \mathbf{s}=(s \sin \kappa, 0, s \cos \kappa), \quad \mathbf{s}^{*}=(s \sin \kappa, 0$, $-s \cos \kappa), \quad \boldsymbol{\alpha}(s)=-\omega / 2 \log \delta(0, s \sin \kappa, 0)$. The first two terms in Eq. (1) can be recognized as line distributions of Stokeslets placed at $\mathbf{s}$ and its image $\mathbf{s}^{*}$ in the lower half space. The remaining terms are built from higher order singularities, namely, the Stokes doublet and the point-source quadripole $[8,10]$. Flagellated organisms have been modeled using slender body theory $[14,15]$, but unlike these studies our model provides exact formulas which require no numerical integration to evaluate the flow.

\section{MACROSCALE EXPERIMENT}

We describe experiments conducted at both macro- and microscopic scales. In both cases magnetically permeable rods are driven by magnetic fields to pin one end of the rod 
to a horizontal planar surface and cause a conical motion of the rod about an approximately vertical axis. We stress that the motion of the rod is precession and not just rotation about one of its axes. The rods are immersed in viscous fluids containing visible marker particles to enable optical tracking of the fluid motion. The fluid viscosities and precession frequencies are such that a Reynolds number of 0.002 was never exceeded. The macroscopic rotational mixing experiment (RMX) rods are of the order of $1 \mathrm{~cm}$ long and $0.2-1 \mathrm{~mm}$ in diameter, sharpened at one end to provide a well defined pivot point about which to precess. A $30 \mathrm{~cm}$ cubical, clear acrylic tank is filled to $11 \mathrm{~cm}$ with a sucrose solution with a viscosity of $3000 \mathrm{cP}$ and is capped with a removable acrylic top. A strong permanent magnet beneath the tank ( $3000 \mathrm{G}$ at the cone height) is mounted on a motorized turntable with an adjustable offset from the center of rotation. The magnetic field gradient pins the point of the rod to the bottom of the tank, while the magnetic torque of the orbiting magnet causes the rod to precess conically. Marker bubbles, produced in a separate beaker, are selected by syringe to have diameters typically $0.1-0.5 \mathrm{~mm}$ and injected into the RMX tank in desired locations. A Nikon D1 camera is placed in front of the tank to capture the $Y Z$ coordinates of the markers $(0.3 \mathrm{~Hz}$ frame rate), while a Nikon D2 camera is placed above to capture the $X Y$ marker coordinates $(1.0 \mathrm{~Hz}$ frame rate). The magnet turntable is then engaged and adjusted so the rod precesses at a $0.15 \mathrm{~Hz}$ rate. A custom designed video centroid tracking algorithm, developed within the Data Tank programming environment, is used to extract the trajectories of the markers.

\section{NANOSCALE EXPERIMENT}

The nanoscale experiments are conducted using magnetic rods and optical markers in a transparent flow cell mounted on a three dimensional force microscope (3DFM) [16]. The 3DFM applies a dynamic magnetic field to a specimen through a set of pole tips arranged as a hexapole with three poles above and three poles below the plane of the precessing rod. A flow cell $(2 \mathrm{~cm}$ by $1 \mathrm{~cm}$ by $150 \mu \mathrm{m})$ comprises a flow channel bounded by lines of vacuum grease inside double-backed tape spacers, all sandwiched between two microscope cover slips. To provide the friction necessary to keep the magnetic rods from slipping, the bottom cover slip of the flow cell is spin coated with a 1-2 $\mu \mathrm{m}$ layer of PDMS. The magnetic system including the flow cell is placed on an inverted optical microscope where polystyrene microbeads (Polysciences, Inc. $=1.05 \mathrm{~g} / \mathrm{cc}$; Diameter $=0.932 \mu \mathrm{m})$ entrained in the flow are imaged in brightfield mode at $120 \mathrm{~Hz}$ frame rate using a progressive scan Pulnix CCD camera. The NA of the water immersion microscope objective is 1.2 giving a depth of focus of $1-2 \mu \mathrm{m}$. Quasistatic $\mathrm{Z}$ measurements are taken by manually refocusing, with resolution limited by the depth of focus, and data read from the control panel of the Nikon Eclipse TE2000-E. The magnetic nanorods (approximately $200 \mathrm{~nm}$ diameter, $20 \mu \mathrm{m}$ long) consist of Ni90Fe10 alloy electrochemically grown in porous anodized aluminum oxide membrane templates. The rods and markers are dispersed in deionized water $(1.0 \mathrm{cP})$ at

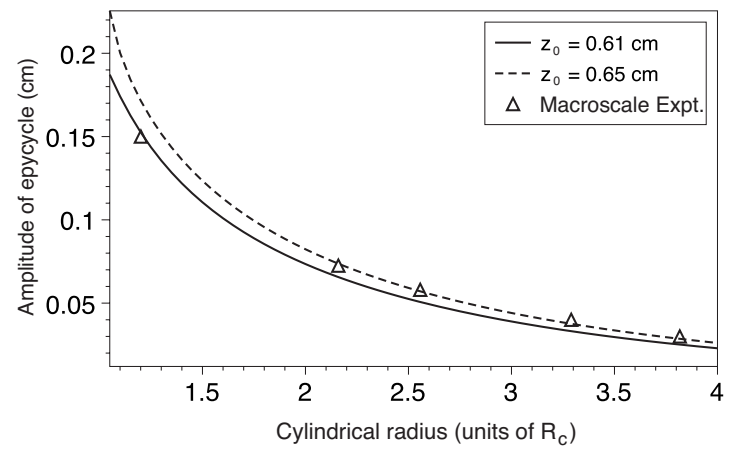

FIG. 2. Theoretical and macroscale experimental radial amplitude. Radial amplitude is the difference between the maximum and minimum of the trajectory cylindrical radius projected on the $z=0$ plane which is taken as the floor of the experimental tank. The amplitude is plotted as a function of the initial cylindrical radius $r_{0}=\sqrt{x_{0}^{2}+y_{0}^{2}}$ (measured in units of cone radius, $R_{c}$ ) of trajectories for two initial vertical positions $z_{0}=0.61 \mathrm{~cm}$ and $z_{0}=0.65 \mathrm{~cm}$ after one rod revolution.

suitable densities, then introduced into the flow cell [17]. The 3DFM magnetic field is energized so as to attract the rods to pin their lower ends to the lower cover slip and align them to the desired cone angle. We note that achieving the desired precession and cone geometry is in itself a challenging inverse problem. This setup is dynamically similar to the RMX geometry in that the Reynolds numbers of both are negligibly small [18]. The rod density is sufficiently low that well isolated rods can be chosen, with suitably distributed beads to measure fluid velocities. A custom designed video centroid tracking program, Spot Tracker [19] with approximately $15 \mathrm{~nm}$ resolution in $X$ and $Y$, is used to extract the orbits of the markers.

\section{THEORETICAL COMPARISON}

\section{A. On the macroscale}

Trajectories in this fluid flow are determined by cone geometry, rod radius, and initial position $\left(x_{0}, y_{0}, z_{0}\right)$ where cone geometry is provided by a cone radius $R_{c}$ and a cone height $H_{c}$. These set a cone angle $\kappa$ and rod length $L$. Several bubble trajectories from the macroscale RMX experiment are compared to the theory presented in Sec. II. The top view of such a comparison is shown in Fig. 1. The trajectories exhibit two superposed motions. The slower one makes a complete orbit about the body at a distance dependent rate. The faster one is epicyclic, related to the precession rate of the rod, with an observable radial amplitude, arclength, vertical fluctuation, and period. The period of an epicycle is a function of cone geometry, rod radius, and initial position. See Fig. 2 caption for radial amplitude definition. Figure 1 shows qualitative agreement between the radial amplitude, arclength, and angle traveled by the epicycles. Figure 2 provides a quantitative comparison of the radial amplitude.

\section{B. On the microscale}

A microbead trajectory from the microscale experiment is shown in Fig. 3 and a comparison to a theoretical trajectory 


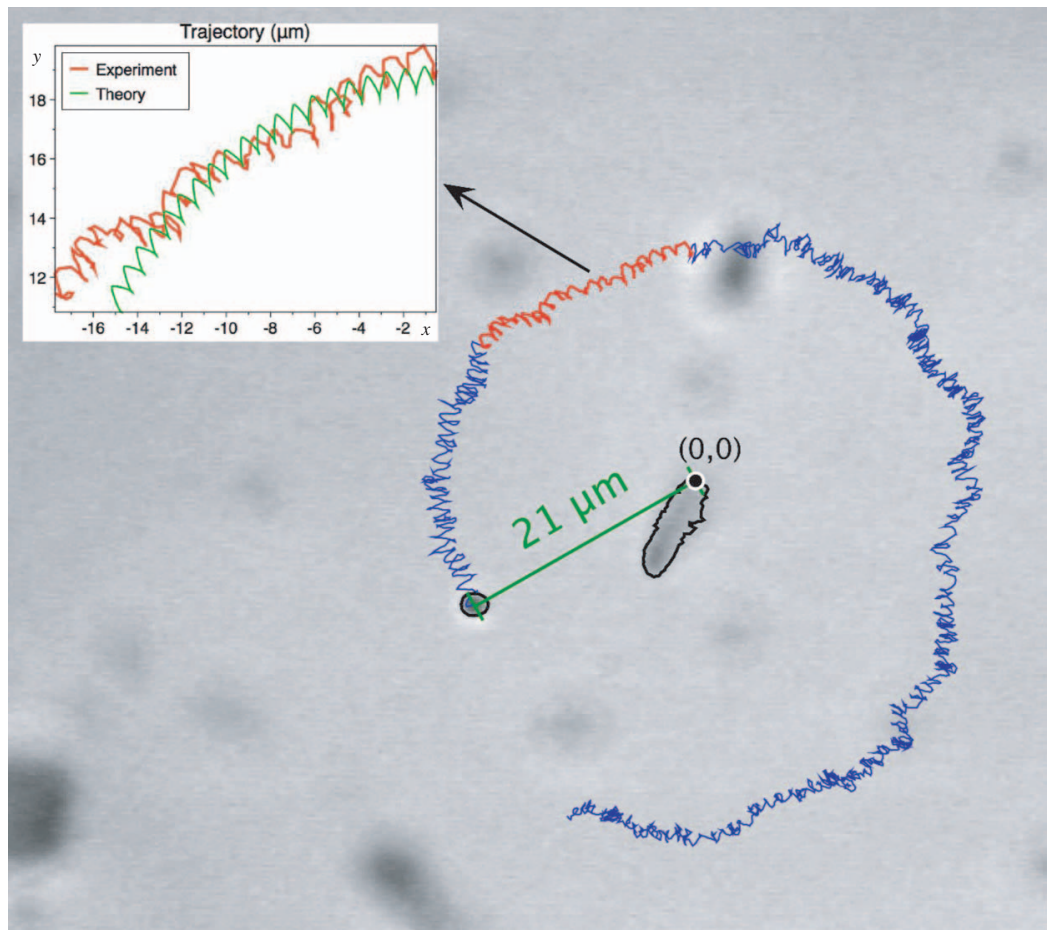

FIG. 3. (Color) Theoretical trajectory compared to a microbead trajectory. The rod base is at the origin and the bead is enclosed by a circular contour. The other darks spots in the figure are debris or beads at different focal heights. The bead trajectory shown is initially at $13.9 \pm 1 \mu \mathrm{m}$ from the floor of the flow cell, the cone radius is $R_{c}=9 \mu \mathrm{m}$, the cone height $H_{c}=9.75 \pm 1 \mu \mathrm{m}$, and the rod rotates counterclockwise at $5 \mathrm{~Hz}$. A comparison to a theoretical trajectory for a portion of the experiment is given in the inset. The theoretical trajectory is computed as in Fig. 1 with $R_{c}=9 \mu \mathrm{m}$ and $H_{c}=9.75 \mu \mathrm{m}$. The initial vertical position is set at $z_{0}=12.9 \mu \mathrm{m}$ and the rod diameter is set to $400 \mathrm{~nm}$.

is given in the inset for a portion of the experiment. Brownian effects are clearly visible in the beads when the rod is held motionless. To obtain averaged statistics, we study power spectra of the cylindrical radius, $\sqrt{x^{2}(t)+y^{2}(t)}$, averaged over a small frequency band about the frequency of the $\operatorname{rod} \omega=5 \mathrm{~Hz}$. The comparison of this statistic is shown in Fig. 4 where the bandwidth is $5 \pm 0.5 \mathrm{~Hz}$. The agreement is strong in the near field, but deteriorates at far field. Although care is taken to find clean isolated rods there may exist rod shape anomalies or precessing rods well outside the field of view contributing to the flow.

Finally, the distinction between macroscale and microscale is clearly the emergence of a strong thermal component of the motion at the microscale. Preliminary analysis indicates that the proper incorporation of these Brownian effects via Langevin dynamics does not produce a satisfactory comparison with the data. Further study is clearly merited upon this point.

\section{CONCLUSIONS}

We performed a quantified comparison of a complete hydrodynamic theory with experiments at both micro- and macroscales, for the fluid motion induced by a precessing rod of interest in biophysics. Future directions will account for flexibility, complex rheology and different geometric boundary forcing.

\section{ACKNOWLEDGMENTS}

Research was supported by NSF DMS-0104329, NSF DMS-0509423, NSF DMS-0308687, NSF DMS-SCREMS 042241, RTG NSF DMS-0502266, NIH P41-EB002025, NIH R01EB000761-01, and NASA URETI on BioInspired

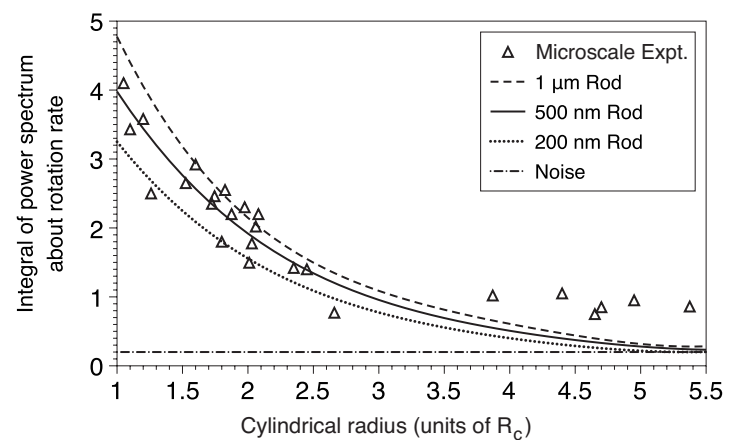

FIG. 4. A measure of theoretical and experimental radial amplitude on the microscale as provided by the power spectrum statistic (see text) as a function of radius. The theoretical predictions of this statistic are provided over rod diameters which vary from $200 \mathrm{~nm}$, $500 \mathrm{~nm}$, and $1 \mu \mathrm{m}$ for an initial vertical position $z_{0}=12.9 \mu \mathrm{m}$. The reference value of this statistic on microbead trajectories in free diffusion is provided as the constant value shown, and is generally much smaller than the actuating data. 
Materials NCC-1-02037. A.B. and J.T were partially supported by NSF DMS-0308687, RTG NSF DMS-0502266, and the UNC office of Undergraduate Research. The tabletop experimental work was supported by NSF DMS-0308687, and the nanoscale experimental work was supported by NIH P41-EB002025 and NIH R01EB000761-01. We thank David Adalsteinsson with tracking using his Data Tank program and Russ Taylor with his Spot Tracker program.
[1] S. Nonaka, Y. Tanaka, Y. Okada, S. Takeda, M. Kido, and N. Hirokawa, Cell 95, 829 (1998).

[2] J. H. E. Cartwright, O. Piro, and I. Tuval, Proc. Natl. Acad. Sci. U.S.A. 101, 7234 (2004).

[3] G. R. Fulford and J. R. Blake, J. Theor. Biol. 121, 381 (1986).

[4] H. Matsui, S. H. Randell, S. W. Peretti, C. W. Davis, and R. C. Boucher, J. Clin. Invest. 102, 1125 (1998).

[5] G. J. Hancock, Proc. R. Soc. London, Ser. A 217, 96 (1953).

[6] E. Lauga, M. Brenner, and H. Stone, Handbook of Experimental Fluid Dynamics (Springer, New York, 2007).

[7] Y. Zhu and S. Granick, Phys. Rev. Lett. 87, 096105 (2001).

[8] J. R. Blake, Proc. Cambridge Philos. Soc. 70, 303 (1971).

[9] G. K. Batchelor, J. Fluid Mech. 44, 419 (1970).

[10] C. Pozrikidis, Introduction to Theoretical and Computational Fluid Dynamics (Oxford University Press, New York, 1997).

[11] R. Camassa, T. J. Leiterman, and R. M. McLaughlin (unpublished).
[12] R. Camassa, T. J. Leiterman, and R. M. McLaughlin (unpublished).

[13] T. J. Leiterman, Ph.D. thesis, University of North Carolina, 2006.

[14] B. A. A. Orme, J. R. Blake, and S. R. Otto, J. Fluid Mech. 475, 333 (2003).

[15] J. J. L Higdon, J. Fluid Mech. 94, 305 (1979).

[16] J. K. Fisher, J. Cribb, K. V. Desai, L. Vicci, B. Wilde, K. Keller, R. M. Taylor, J. Hasse, K. Bloom, E. T. O’Brien, and R. Superfine, Rev. Sci. Instrum. 77, 023702 (2006).

[17] H. Jing, Ph.D. thesis, University of North Carolina, 2006.

[18] To verify that finite volume (Faxen) corrections are not required, we repeated the macroscale experiment with a large dynamically similar sphere as used in the microscale, and observed no significant difference in the radial amplitude as compared with the bubble measurements.

[19] R. M Taylor, www.cs.unc.edu/cismm/download/spottracker/ video_spot_tracker.html (2005). 